\title{
Genetic Variants Are Not Rare in ICD Candidates with Dilated Cardiomyopathy: Time for Next-Generation Sequencing?
}

\author{
Alexandra Sousa $\mathbb{D}^{1},{ }^{1,2,3}$ Paulo Canedo, ${ }^{4}$ Manuel Campelo, ${ }^{1,2,5}$ Brenda Moura, ${ }^{6}$ Sérgio Leite, ${ }^{7}$ \\ Márcia Baixia, ${ }^{4}$ Adriana Belo, ${ }^{8}$ Francisco Rocha-Gonçalves, ${ }^{1,4}$ José Carlos Machado, ${ }^{4}$ \\ José Silva-Cardoso, ${ }^{1,2,5}$ Elisabete Martins $\mathbb{D}^{1,4,5}$ and FATIMA Investigators ${ }^{8}$ \\ ${ }^{1}$ Department of Medicine, Faculty of Medicine, Alameda Prof. Hernâni Monteiro, 4200-319 Porto, Portugal \\ ${ }^{2}$ Cintesis-Center for Research in Health Technologies and Services, Rua Dr. Plácido da Costa, 4200-450 Porto, Portugal \\ ${ }^{3}$ Department of Cardiology, Santa Maria Maior Hospital, Campo da República, Apartado 181, 4754-909 Barcelos, Portugal \\ ${ }^{4}$ i3S-Institute for Research and Innovation in Health, Rua Alfredo Allen, 4200-135 Porto, Portugal \\ ${ }^{5}$ Department of Cardiology, Centro Hospitalar Universitário de São João, E.P.E., Alameda Prof. Hernâni Monteiro, \\ 4200-319 Porto, Portugal \\ ${ }^{6}$ Department of Cardiology, Hospital das Forças Armadas - Pólo Porto, Av. Da Boavista, 4050-113 Porto, Portugal \\ ${ }^{7}$ Department of Cardiology, Alto Ave Hospital Center-Guimarães Unity, Rua dos Cutileiros 114, Creixomil, \\ 4835-044 Guimarães, Portugal \\ ${ }^{8}$ National Center for Data Collection in Cardiology, Rua de Olivença $n^{\circ} 11,7^{\circ}$ Piso, Sala 701, 3000-306 Coimbra, Portugal \\ Correspondence should be addressed to Alexandra Sousa; xanasousa81@gmail.com
}

Received 23 September 2018; Revised 20 November 2018; Accepted 12 February 2019; Published 24 April 2019

Academic Editor: Mariantonietta Cicoira

Copyright (c) 2019 Alexandra Sousa et al. This is an open access article distributed under the Creative Commons Attribution License, which permits unrestricted use, distribution, and reproduction in any medium, provided the original work is properly cited.

\begin{abstract}
Background. Sudden cardiac death (SCD) risk stratification in dilated cardiomyopathy (DCM) has been based on left ventricular ejection fraction (LVEF), even though SCD may occur with LVEF $>35 \%$. Family history of unexplained SCD, especially in the young, raises concern about potential inheritable risk factors. It remains largely unknown how genetic tests can be integrated into clinical practice, particularly in the selection of implantable cardioverter defibrillator (ICD) candidates. We aimed to assess the diagnostic yield of genetic testing in DCM patients with a class I recommendation for ICD implantation, based on current guidelines. Methods. We included ambulatory stable adult patients with idiopathic or familial DCM with previously implanted ICD. Molecular analysis included 15 genes (LMNA, MYH7, MYBPC3, TNNT2, ACTC1, TPM1, CSRP3, TCAP, SGCD, PLN, MYL2, MYL3, TNNI3, TAZ, and LDB3) using next-generation sequencing. Results. We evaluated 21 patients, 12 (57\%) males and 9 (43\%) with familial DCM, including 3 (14\%) with a family history of premature unexplained SCD. Mean age at DCM diagnosis was $40 \pm 2$ years, and mean age at ICD implantation was $50 \pm 12$ years. $L V E F$ was $27 \pm 9 \%$, and LV end-diastolic diameter was $65 \pm 7 \mathrm{~mm}$. Genetic variants were found in six (29\%) patients, occurring in 5 genes: TPM1, TNNT2, MYH7, PLN, and MYBPC3. The majority were classified as variants of uncertain significance. Family history of SCD was present in both patients with PLN variants. Conclusion. In patients with DCM and ICD, genetic variants could be identified in a significant proportion of patients in several genes, highlighting the potential role of genetics in DCM SCD risk stratification.
\end{abstract}

\section{Introduction}

Sudden cardiac death (SCD) is responsible for a significant number $(30 \%)$ of deaths in patients with DCM $[1,2]$, and implantable cardioverter defibrillator (ICD) devices can reduce this risk [3].
Currently, the preferred clinical tool to estimate SCD risk and decide about ICD implantation for primary prevention is the presence of $L V$ ejection fraction (LVEF) value $\leq 35 \%$, after heart failure medical therapy uptitration [1]. However, risk prediction of SCD in DCM patients is far from being ideal. Most of the episodes of SCD continue to occur despite LVEF 
values higher than $35 \%$ and, most recently, the DANISH trial showed no long-term mortality benefit for prophylactic ICD implantation, in patients with symptomatic nonischemic heart failure with reduced ejection fraction [4].

The diversity of etiologies and heterogeneity of arrhythmogenic mechanisms can explain the difficulty in predicting SCD risk in DCM patients.

A frequently unrecognized subgroup of patients is that with genetic DCM. Genetic factors have been associated with higher arrhythmic risk [5], but in most patients, genetic tests are not performed before ICD implantation. According to Heart Rhythm Society (HRS) and the European Heart Rhythm Association (EHRA), genetic screening is recommended when there is evidence of DCM and significant cardiac conduction disease and/or a family history of unexplained premature sudden cardiac death. These characteristics are considered as "red flags" for LMNA and SCN5A associated disease [6].

Nowadays, molecular diagnosis is performed using nextgeneration sequencing (NGS) that allows several genes to be sequenced simultaneously, with decreased costs and turnaround times. This technical advantage is particularly useful in DCM, a disease with a high genetic and allelic heterogeneity, with 60 to $90 \%$ of causal variants found in only single families [7]. The main difficulty related to the higher yield of genetic variants obtained with NGS is the ascription of pathogenicity for most variants [8]. Crossing data from different genetic databases, containing clinical and laboratory information of DCM patients, is of utmost importance for this purpose, and also to highlight the usefulness of genetic tests in arrhythmic risk prediction algorithms.

In our study, we aimed to assess the diagnostic yield of genetic analysis using NGS in DCM patients with a priori class I recommendation for ICD implantation, based on the European Society of Cardiology guidelines [1].

\section{Materials and Methods}

2.1. Population. A multicentric study of adult unrelated ambulatory patients (stable in NYHA I or II for at least 6month) with idiopathic DCM (age $\leq 50$ years) or familial DCM (irrespective of age), with a class I indication for ICD implantation [9], was included in the study. DCM was diagnosed according to the Working Group on Myocardial and Pericardial Disease of the ESC criteria [10]. DCM was considered idiopathic in the absence of evidence for a secondary or acquired cause of the disease. Familial disease was established when idiopathic DCM was present in more than one family member or when unexplained SCD occurred in any first-degree relative under the age of 35 years $[11,12]$. Nonfamilial disease was considered in the remaining cases, after a complete and detailed questionnaire on the patient family history and clinical, electrocardiographic, and echocardiographic evaluation of first-degree relatives.

This study is a subanalysis of FATIMA (Portuguese study of familial dilated cardiomyopathies) and general clinical assessment, and cardiological investigations as well as exclusion criteria (any possible secondary or acquired cause of the disease) have been previously described [11]. We also excluded patients considered for or on the waiting list for heart transplantation. Cardiovascular events, such as cardiovascular death and ventricular arrhythmic episodes, were collected in each clinical visit by anamnesis and consult of clinical records.

2.2. Next-Generation Sequencing. A peripheral blood sample was obtained for molecular analysis from each patient. Screening of variants in a panel of 15 genes-lamin A/C (LMNA), beta-myosin heavy chain (MYH7), myosinbinding protein $\mathrm{C}$ (MYBPC3), troponin-T (TNNT2), alpha-actin (ACTA1), alpha-tropomyosin (TPM1), cysteinerich protein 3 (CSRP3), titin-cap (TCAP), sarcoglycan delta $(S G C D)$ and phospholamban $(P L N)$, regulatory and essential light chains (MYL2 and MYL3), troponin-I (TNNI3), tafazzin (TAZ), and ZASP/Cypher (LDB3) - was performed in all patients, using NGS with a minimum of 30 -fold coverage and guaranteed $100 \%$ horizontal coverage for the coding sequencing and flanking exon/intron regions. Sanger sequencing was used to validate the identified variants and to provide additional coverage for regions of the panel with less than 30-fold coverage. Genes were selected, and the targeted gene panel was designed in 2010, based on previously identified variants in DCM patients at the time.

For the screening of variants in the selected genes, primers were designed for all coding exons, covering exon/ intron boundaries using freely available software Primer3. No known variants were present in the primer sequences (dbSNP built 130). A multiplex PCR-based strategy was used to reduce the number of amplification reactions (primer sequences are provided upon request). Multiplex PCR reactions were performed following the QIAGEN Multiplex PCR Kit protocol (Qiagen, Hilden, Germany).

2.2.1. Library and Template Preparation. Sample quality of patient genomic DNA was evaluated by gel electrophoresis and quantified using Qubit dsDNA HS Assay Kit (Life Technologies). A total of $50 \mathrm{ng}$ of genomic DNA was used in each multiplex PCR reaction. Library preparation was performed using the protocol Ion Xpress Plus gDNA and amplicon library preparation (PN4471989 Rev. C). Library quantification was performed using the Qubit dsDNA HS assay. All libraries were diluted to the same concentration and pooled to ensure an equal representation of the different samples. The diluted and combined libraries were subjected to amplification by emulsion PCR using Ion Template OT 2 200 Kit (Life Technologies) on an Ion OneTouch 2 instrument (Life Technologies) according to the manufacturer's protocol. Enrichment of template ion sphere particles was performed using the Ion OneTouch 2 enrichment system (Life Technologies) (Figure 1).

2.2.2. Semiconductor Sequencing and Data Analysis. Sequencing was carried out on an Ion PGM system based on semiconductor technology. The Ion Sequencing Kit v2.0 (Life Technologies) was used to perform sequencing runs, 


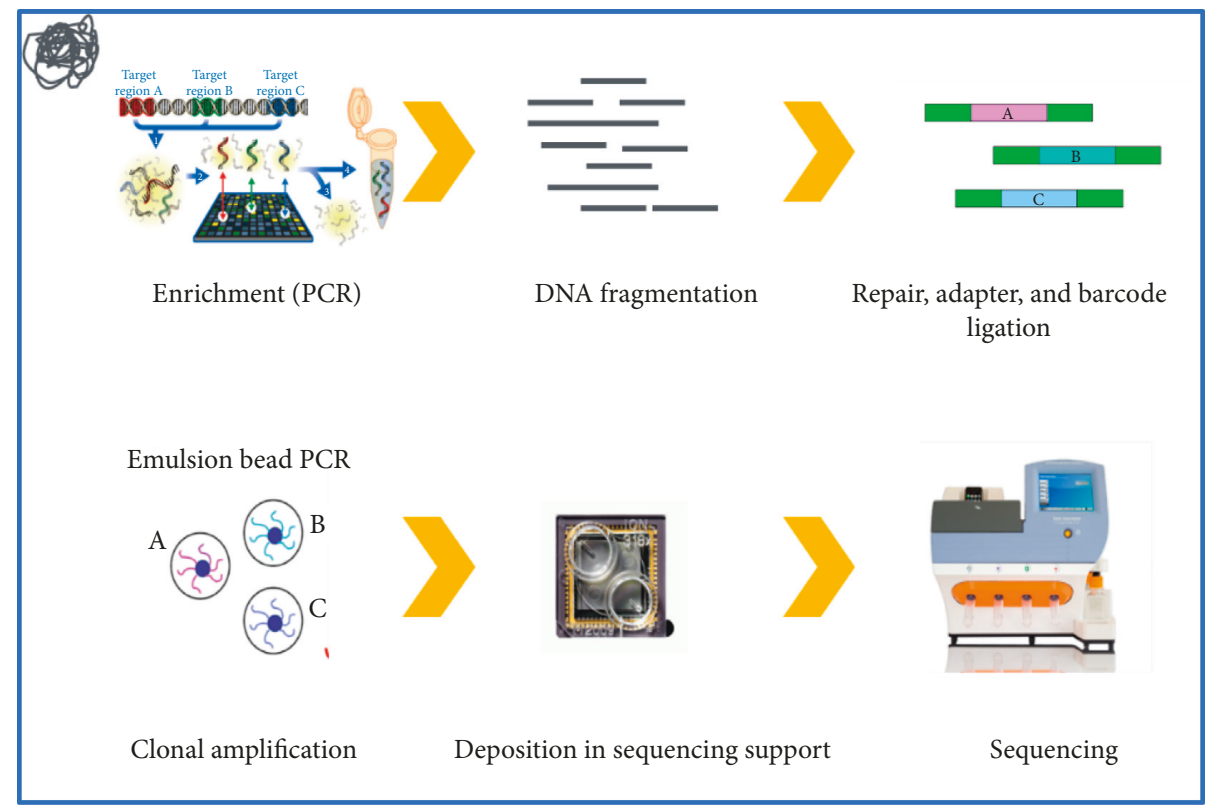

FIgURE 1: Molecular analysis: library and template preparation.

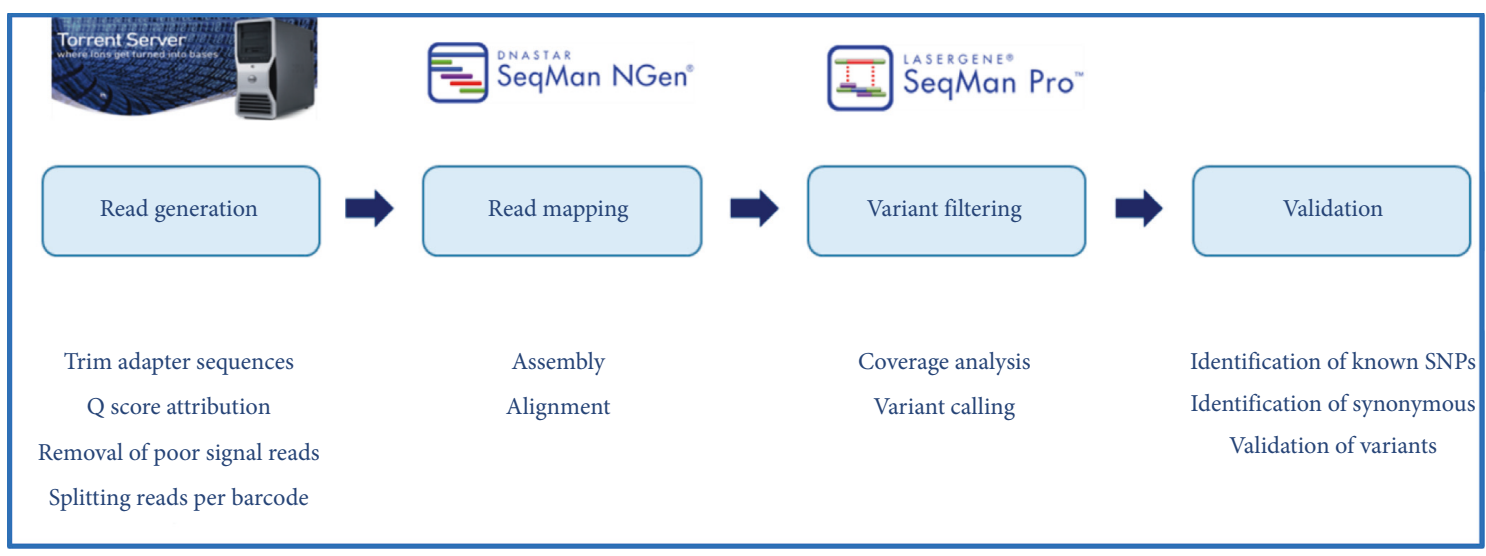

FIGURE 2: Molecular analysis: bioinformatic pipeline.

following the manufacturer's recommended protocols. Data from the PGM runs were processed using the Ion Torrent platform-specific pipeline software Torrent Suite v4.2 (Life Technologies) to generate sequence reads, trim adapter sequences, filter and remove poor signal reads, and split the reads according to the barcode. Reads assembly was performed with SeqMan NGen v4.1 (DNAStar, Madison, Wisconsin) using the FastQ files containing sequence reads and the template references adjusted for the covered amplicons. SeqMan Pro v10 (DNAStar) was used as a postassembly analysis tool for the analysis of overall amplicon coverage, individual base depth of coverage (only considered if coverage has a value of, at least, 30), and variant identification. A filter for the coding sequencing variants (CDS) was applied to the SNP report, for each case. All rare nonsynonymous variants identified $(\mathrm{MAF}<0.01)$ were independently confirmed by Sanger sequencing (Figure 2).

From there, each variant was interpreted and pathogenicity was assessed in accordance with the American College of Medical Genetics and Genomics [8], by comparisons with populational and disease databases, where genetic variants have been previously described, and predictive bioinformatics models, familial segregation analysis, and functional studies when available. They were classified in "pathogenic," "likely pathogenic," "of uncertain significance," "likely benign," and "benign."

2.3. Ethical Approval. The study protocol conforms to the ethical guidelines of the Declaration of Helsinki, 1975, as reflected in a priori approval by the local ethics committees for human research. Informed consent was obtained from each patient.

\section{Results}

We evaluated 21 patients, 12 (57\%) male, 9 (43\%) with familial DCM, including 3 (14\%) with a family history of 
premature unexplained SCD. Mean age at DCM diagnosis was $40 \pm 12$ years, and the mean age at ICD implantation was $50 \pm 12$ years. Echocardiographic LVEF was $27 \pm 9 \%$, LV end-diastolic diameter $65 \pm 7 \mathrm{~mm}$, and 7 (33\%) patients also presented right ventricular dysfunction. Cardiac magnetic resonance $(\mathrm{CMR})$ was only performed in seven $(33 \%)$ patients, since the previous implantation of ICD precluded its execution. In these patients, LVEF was $28 \pm 10 \%$, LV enddiastolic volume was $144 \pm 26 \mathrm{~mL} / \mathrm{m}^{2}$, and two patients (29\%) presented late gadolinium enhancement. Left bundle branch block was present in ten (48\%) patients, and six patients (35\%) had documentation of nonsustained ventricular tachycardia on the 24-holter recording. No patient had evidence of second and/or third atrioventricular block. Two patients had elevated plasma creatinine kinase. Further patients' characterization is displayed in Table 1.

Rare genetic variants were found in six (28\%) of these patients, occurring in 5 different genes: TPM1, TNNT2, MYH7, PLN, and MYBPC3. Tables 2 and 3 show the clinical characteristics of those patients and genetic variant classification, respectively. The pedigree and the ECG of a PLN carrier are illustrated in Figure 3.

The TPM1 variant, c.758T > C p.(Ile253Thr), was absent from controls (ExAC; 1000 genomes) and from diseasespecific databases. In silico analysis predicts it to be diseasecausing and probably damaging to the protein structure/ function. To our knowledge, this residue is conserved throughout evolution. No other variants have been reported in association with disease in nearby codons.

The TNNT2 variant, c.517C > T p.(Arg173Trp), was absent from controls (ExAC; 1000 genomes), and it has four entries in ClinVar as pathogenic/likely pathogenic. This variant is a nonconservative amino acid substitution, which is likely to impact secondary protein structure as these residues differ in polarity, charge, size, and/or other properties, which is supported by in silico analysis. This substitution occurs at a position in which amino acids with similar properties to arginine are tolerated across species. Another variant in the same residue (Arg173Gln) as well as variants in nearby residues (Glu163Lys, Ala172Ser, and Ser179Phe) have been reported at HGMD in association with cardiomyopathy. Cardiomyocytes generated from induced pluripotent stem cells from patients of a family harboring Arg173Trp exhibited altered $\mathrm{Ca}^{2+}$ handling and impaired myofilament regulation [22]. In [23], this variant segregated with all affected family members in two families. Also, a patient with a borderline phenotype did not harbor the variant and did not progress in over more than 20 years of follow-up [23].

The MYH7 variant, c.5623G > T p.(Val1875Phe), was absent from controls (ExAC; 1000 genomes) and from disease-specific databases. In silico analysis predicts it to be disease-causing and probably damaging due to the protein structure/function. To our knowledge, this is a conservative amino acid change, and this residue is conserved throughout evolution. No other variants have been reported in association with disease in nearby codons. A variant in the same
TABle 1: Patients' characteristics.

\begin{tabular}{|c|c|}
\hline Characteristics & $\begin{array}{l}\text { DCM patients } \\
\quad(n=21)\end{array}$ \\
\hline \multicolumn{2}{|l|}{ Gender, $n(\%)$} \\
\hline Male & $12(57)$ \\
\hline Female & $9(43)$ \\
\hline Age at diagnosis, years $($ mean $\pm \mathrm{SD})$ & $40 \pm 12$ \\
\hline $\begin{array}{l}\text { Age at ICD implantation, years } \\
(\text { mean } \pm \mathrm{SD})\end{array}$ & $50 \pm 12$ \\
\hline Familial DCM, $n(\%)$ & $9(43)$ \\
\hline Idiopathic DCM, $n(\%)$ & $12(57)$ \\
\hline \multicolumn{2}{|l|}{ Family history, $n(\%)$} \\
\hline Heart failure-related death & $7(33)$ \\
\hline Sudden death & $3(14)$ \\
\hline \multicolumn{2}{|l|}{ Clinical presentation, $n(\%)$} \\
\hline Heart failure symptoms & $7(33)$ \\
\hline Syncope or arrhythmia & $3(14)$ \\
\hline Chest pain & $5(24)$ \\
\hline Others* & $1(5)$ \\
\hline \multicolumn{2}{|l|}{ NYHA functional class, $n(\%)$} \\
\hline $\mathrm{I}$ & $6(28)$ \\
\hline II & $13(62)$ \\
\hline III & $1(5)$ \\
\hline IV & $1(5)$ \\
\hline Previous hospitalizations, $n(\%)$ & $13(62)$ \\
\hline Heart failure-related & $10(48)$ \\
\hline Arrhythmic causes & $7(33)$ \\
\hline Others $^{\dagger}$ & $1(5)$ \\
\hline \multicolumn{2}{|l|}{ ECG data, $n(\%)$} \\
\hline $\mathrm{AF} /$ atrial flutter & $4(19)$ \\
\hline LBBB & $10(48)$ \\
\hline PVC & $5(24)$ \\
\hline NSVT & $6(35)$ \\
\hline \multicolumn{2}{|l|}{ Echocardiographic data } \\
\hline LVEDD $(\mathrm{mm})($ mean $\pm \mathrm{SD})$ & $65 \pm 7$ \\
\hline $\operatorname{LVEF}(\%)($ mean $\pm \mathrm{SD})$ & $27 \pm 9$ \\
\hline RV impairment, $n(\%)$ & $7(33)$ \\
\hline CMR data, $n(\%)$ & $7(33)$ \\
\hline $\operatorname{LVEDV}\left(\mathrm{mL} / \mathrm{m}^{2}\right)($ mean $\pm \mathrm{SD})$ & $144 \pm 26$ \\
\hline $\operatorname{LVEF}(\%)($ mean \pm SD) & $28 \pm 10$ \\
\hline LGE, $n(\%)$ & $2(28)$ \\
\hline \multicolumn{2}{|l|}{ Medical therapy, $n(\%)$} \\
\hline ACEI/ARB & $19(90)$ \\
\hline Beta-blockers & $18(86)$ \\
\hline MRA & $14(67)$ \\
\hline Diuretic & $12(57)$ \\
\hline Digoxin & $6(28)$ \\
\hline Ivabradine & $2(10)$ \\
\hline
\end{tabular}

${ }^{*}$ Asymptomatic left ventricular dysfunction diagnosed on medical sportive examination. ${ }^{\dagger}$ Elective hospitalization for atrial flutter ablation. ACEI: angiotensin converting enzyme inhibitor; AF: atrial fibrillation; ARB: angiotensin II receptor blocker; CMR: cardiac magnetic resonance; DCM: dilated cardiomyopathy; ECG: electrocardiogram; ICD: implantable cardioverter defibrillator; LBBB: left bundle branch block; LGE: late gadolinium enhancement; LVEDD: left ventricular end-diastolic diameter; LVEDV: left ventricular end-diastolic volume; LVEF: left ventricular ejection fraction; MRA: mineralocorticoid receptor antagonist; NSVT: nonsustained ventricular tachycardia; NYHA: New York Heart Association; PVC: premature ventricular contraction; RV: right ventricle; SD: standard deviation. 
TABLE 2: Clinical characteristics of patients with genetic variants.

\begin{tabular}{lcccccccccc}
\hline Case & Gender & Age & $\begin{array}{c}\text { Age at } \\
\text { diagnosis }\end{array}$ & $\begin{array}{c}\text { NYHA } \\
\text { class }\end{array}$ & Etiology & $\begin{array}{c}\text { ECG-rhythm } \\
\text { disturbances }\end{array}$ & $\begin{array}{c}\text { ECG-conduction } \\
\text { disturbances }\end{array}$ & $\begin{array}{c}\text { LVEF } \\
(\%)\end{array}$ & $\begin{array}{c}\text { LVED } \\
(\mathrm{mm})\end{array}$ & $\begin{array}{c}\text { Affected } \\
\text { gene }\end{array}$ \\
\hline 1 & Female & 40 & 17 & II & IDCM & SR & - & 38 & 61 & TPM1 \\
2 & Male & 53 & 38 & II & IDCM & SR & LBBB & 38 & 63 & TNNT2 \\
3 & Male & 55 & 53 & II & IDCM $^{*}$ & SR & LBBB & 39 & $56^{\dagger}$ & MYH7 \\
4 & Female & 56 & 47 & I & FDCM $^{\ddagger}$ & SR & - & 37 & 68 & PLN \\
5 & Male & 53 & 41 & II & FDCM $^{\ddagger}$ & SR & LBBB & 33 & 62 & PLN \\
6 & Female & 72 & 58 & II & FDCM & AF & LF & 71 & MYBPC3 \\
\hline
\end{tabular}

FDCM: familial dilated cardiomyopathy; IDCM: idiopathic dilated cardiomyopathy; LBBB: left bundle branch block; LVED: left ventricular end-diastolic diameter; LVEF: left ventricular ejection fraction; SR: sinus rhythm; ${ }^{*}$ serum creatine kinase elevation; ${ }^{\dagger}$ left ventricular hypertrabeculation/noncompaction; * sudden death in relatives.

residue (Val1875Ala) has an entry in ClinVar, as uncertain significance.

As for the PLN variants, only one missense variant is reported as pathogenic in ClinVar (Arg9Cys). The variant c.61C > A p.(Pro21Thr) is present in very low frequency in controls (ExAC 0.00006 (A)) and, despite being a nonconservative change, the in silico analysis does not have concordant results. The variant c.23C $>\mathrm{T}$ p.(Thr8Ile) was absent from controls (ExAC; 1000 genomes) and from disease-specific databases. In silico analysis predicts it to be disease-causing and probably damaging to the protein structure/function. A variant in the same residue (Thr8Pro) has an entry in ClinVar, as uncertain significance. Also, despite being a nonconservative change, the in silico analysis does not have concordant results.

The MYBPC3 variant c. $1226+6 \mathrm{~T}>\mathrm{C}$ is present in very low frequency in controls (ExAC $0.00006(\mathrm{G})$ ), and it has one entry in ClinVar as uncertain significance. This variant is not located in a canonical splice site and therefore is a rare intronic change with uncertain impact on splicing.

When comparing patients with and without a rare variant, there were no significant differences in main demographic, clinical, and electrocardiographic characteristics, although those with a positive molecular study presented higher LVEF ( $34 \pm 7 \%$ vs. $24 \pm 8 \%, p=0.024$ ) (Table 4).

After a mean follow-up of $21 \pm 8$ months (range 5-30), 3 (14\%) patients experienced adverse events. Two patients were hospitalized because of heart failure, and one of them ultimately died from pump failure; the other presented ventricular arrhythmia, namely, a nonsyncopal monomorphic ventricular tachycardia (eight months later underwent heart transplantation). The patient who died from heart failure had the MYBPC 3 variant c. $1226+6 \mathrm{~T}>\mathrm{C}$, and the remaining had a negative molecular study.

\section{Discussion}

In our DCM patients with a class I indication for ICD implantation, using NGS methodology for genetic analysis, we found a non-negligible number of variants in different genes. Most of the identified variants, although predicted to be functionally significant, were classified as of uncertain significance mainly because the evaluation of patients' families did not allow conclusions about segregation and because half of them were novel variants, not previously described (MYH7 p.(Val1875Phe), TPM1 p.(Ile253Thr) and PLN p. (Thr8Ile)). Only the TNNT2 variant p.(Arg173Trp) was previously described in DCM families $[20,23]$. The MYBPC3 variant c. $1226+6 \mathrm{~T}>\mathrm{C}$ was previously described in association with hypertrophic cardiomyopathy [20].

In general, there were no major clinical differences between patients with or without genetic variants in the analyzed genes. Interestingly, patients with positive molecular study presented a higher LVEF. This could be related to a higher proportion of patients with ICD implanted in a context of secondary prevention of sudden cardiac death or we could hypothesize that the involved genes might be somehow related to left ventricular reverse remodelling as has been shown recently for titin [24] and other genes [25]. Also, the presence of a genetic variant did not influence the occurrence of an adverse event, although the small number of participants and events limits our conclusions. Even so, these results are interesting, as they put in perspective the potential role of genetics in SCD risk stratification.

DCM genetics has been previously assessed in patients with life-threatening arrhythmias and analyzed in few larger genotype-phenotype correlation studies, including the INTERHEART study, where $27 \%$ received ICD implantation [26]. SCD risk has been particularly associated with $L M N A$ variants, but also in other genes, such as PLN, SCN5A, and FLNC and some desmosomal genes [5, 27, 28].

In our patients, we did not find any variant in the LMNA gene, but this could be partially related to the fact that no patient presented evidence of atrioventricular conduction block on ECG and no patient considered for or on the waiting list for heart transplantation has been included (even though almost half of our patients presented left bundle branch block and 35\% presented nonsustained ventricular tachycardia, features also common in LMNA pathogenic variants carriers with DCM) [5]. On the other hand, two of our three patients with a family history of SCD presented PLN gene variants, one of which is a novel variant (Thr8lle).

Phospholamban is a key protein involved in calcium metabolism, regulating SERCA $\mathrm{Ca}^{2+}$ affinity at the sarcoplasmic reticulum. Mutations in PLN can be identified in 2\% of DCM patients [27], and variants in this gene have been particularly associated with arrhythmogenic cardiomyopathies, including right ventricular dysplasia with predominant LV involvement $[29,30]$. The arrhythmogenic mechanisms of 


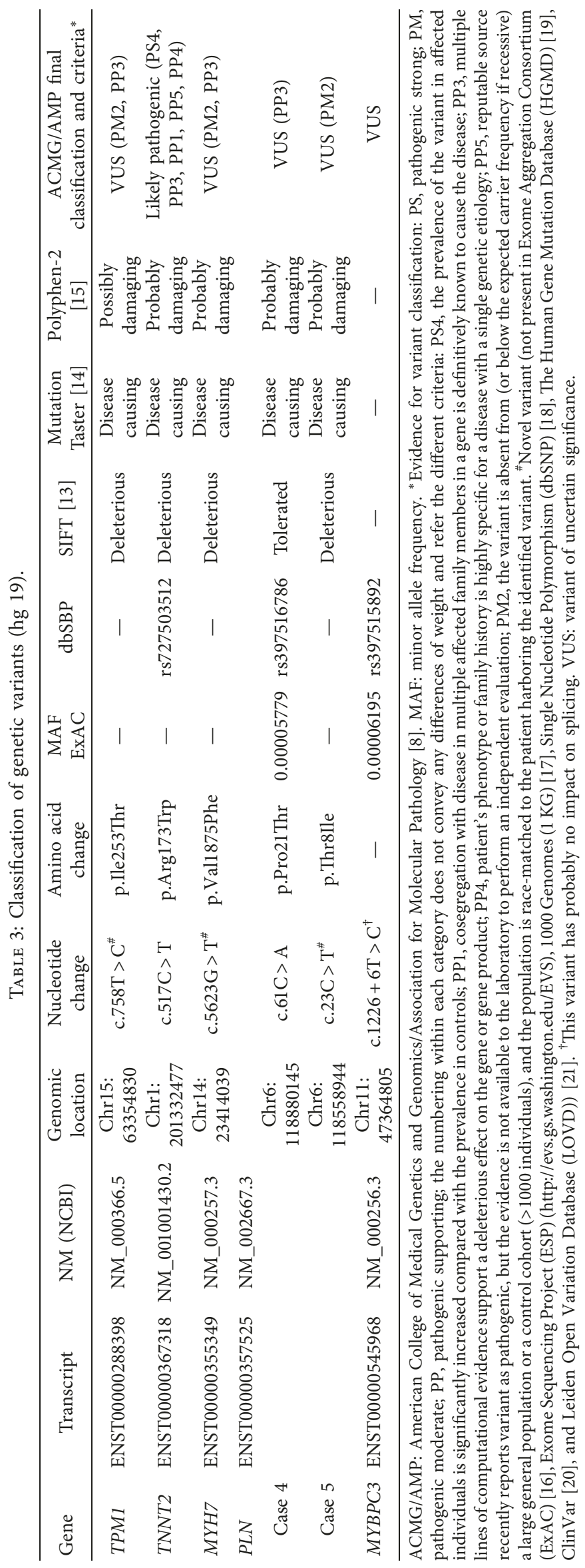




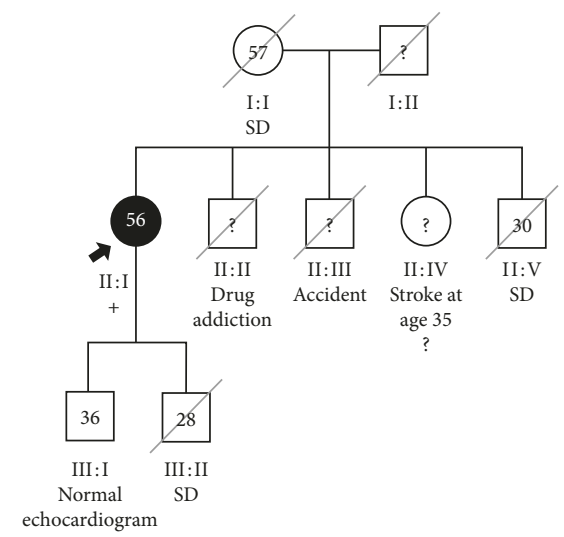

(a)

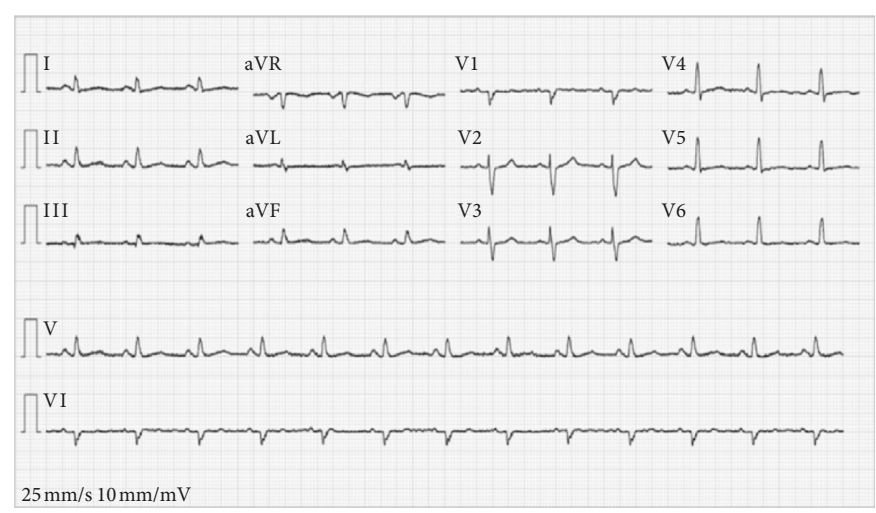

(b)

Figure 3: Pedigree (a) and electrocardiogram (b) of the patient with the p.Pro21Thr phospholamban variant. The electrocardiogram reveals sinus rhythm and a QRS width of $101 \mathrm{~ms}$. Squares: male; circles: female; dark symbol: dilated cardiomyopathy; \pm symbols: presence/absence of PLN variant; numbers inside the symbols: current age; SD: sudden death; "?": inexistent information or availability for clinical/genetic assessment.

TABle 4: Patients' characteristics according to molecular study results.

\begin{tabular}{|c|c|c|c|}
\hline Patient characteristics & No variant $(n=15)$ & Variant-positive $(n=6)$ & $p$ value \\
\hline Age at $d x$, years $($ mean $\pm \mathrm{SD})$ & $40 \pm 11$ & $42 \pm 14$ & 0.633 \\
\hline Age at ICD implantation, years (mean \pm SD) & $50 \pm 12$ & $51 \pm 13$ & 0.872 \\
\hline Male, $n(\%)$ & $9(60)$ & $3(50)$ & 1.000 \\
\hline Familial cases, $n(\%)$ & $6(40)$ & $3(50)$ & 1.000 \\
\hline \multicolumn{4}{|l|}{ Family history, $n(\%)$} \\
\hline Sudden cardiac death & $1(7)$ & $2(33)$ & 0.184 \\
\hline Previous hospitalizations, $n(\%)$ & $9(64)$ & $4(67)$ & 1.000 \\
\hline Heart failure-related & $8(57)$ & $2(33)$ & 0.628 \\
\hline Arrhythmic causes & $4(29)$ & $3(50)$ & 0.613 \\
\hline \multicolumn{4}{|l|}{ NYHA, $n(\%)$} \\
\hline$>\mathrm{I}$ & $10(67)$ & $5(83)$ & 0.802 \\
\hline \multicolumn{4}{|l|}{ ECG data, $n(\%)$} \\
\hline LBBB & $7(47)$ & $3(50)$ & 1.000 \\
\hline Atrial fibrillation & $3(20)$ & $1(17)$ & 1.000 \\
\hline NSVT & $4(36)$ & $2(33)$ & 1.000 \\
\hline PVC & $5(33)$ & $0(0)$ & 0.262 \\
\hline \multicolumn{4}{|l|}{ Echocardiogram data } \\
\hline $\operatorname{LVEDD}(\mathrm{mm})($ mean $\pm \mathrm{SD})$ & $65 \pm 7$ & $63 \pm 6$ & 0.503 \\
\hline $\operatorname{LVEF}(\%)($ mean \pm SD $)$ & $24 \pm 8$ & $34 \pm 7$ & 0.024 \\
\hline RV impairment, $n(\%)$ & $4(31)$ & $3(50)$ & 0.617 \\
\hline
\end{tabular}

ICD: implantable cardioverter defibrillator; LBBB: left bundle branch block; LVEDD: left ventricular end-diastolic diameter; LVEF: left ventricular ejection fraction; NSVT: nonsustained ventricular tachycardia; RV: right ventricle; SD: standard deviation.

PLN variants are largely unknown, but probably they are variable and depend on the variant itself [31]. Mechanisms involving the irreversible superinhibition of sarcoplasmic reticulum ATPase due to the deletion of arginine 14 in PLN gene (Arg14del) have been described in familial DCM with premature sudden cardiac death [32, 33]. Recently, a new hypothesis involving interference with intercalated disc remodelling has been proposed [34]. In both of our patients with PLN variants, arrhythmogenicity could essentially be inferred from family history, particularly in the case 4 , presented in Figure 3. This proband is a woman with the diagnosis of DCM at the age of 47, with mild symptoms of heart failure and documented runs of nonsustained ventricular tachycardia, with history sudden death in three first-degree relatives. Of note, her ECG did not present any distinctive characteristic (only a slight prolongation of the duration of the QRS), unlike the ECG of PLN p.(Arg14del) carriers, which presented strikingly attenuated $\mathrm{R}$ amplitudes, independent of the presence of echocardiographic abnormalities [32, 33]. This particular eletrocardiographic characteristic is apparently related with ventricular fibrosis $[32,33]$. Unfortunately, CMR was not performed in any of our patients before device implantation, an exam that could have added some other important details in phenotypic characterization [35].

In order to improve the knowledge about arrhythmogenesis in DCM and the clinical usefulness of genetics in 
SCD risk stratification, it would be interesting to consider molecular diagnosis as an integrant part of all DCM patients' evaluation, as recommended in recent guidelines on genetic evaluation on cardiomyopathies [36]. This would allow the creation of clinical databases of different sequence variations with information about the clinical profile (including age of onset, disease course, and any unique features and outcomes) along with the variant identified, increasing insights into genotype-phenotype correlations, in a larger group of patients, and permitting continued and extended follow-up of genetically characterized DCM patients.

\section{Limitations}

The major limitation of our work is the small number of patients and the restricting panel of analyzed genes, particularly the noninclusion of titin that accounts for up to $20-25 \%$ of all DCM cases [27], as well as other "arrhythmogenic" genes (when the study was designed, the panel of genes was chosen, given the published overall percentage of dilated cardiomyopathy cases caused by pathogenic variants in each gene, being one of the broader panels available at the time for cardiomyopathies). Even though this study refers to a rather small group of patients and the number of genes analyzed is limited, our results may be a contribution to the better understanding of electric burden in patients with dilated cardiomyopathy who are candidates to ICD implantation.

Another important limitation refers to our inability to differentiate the setting of ICD implantation (primary vs. secondary prevention). Additionally, we did not include patients with ICD considered for or on the waiting list for heart transplantation, because we considered that they represent another particular subgroup of patients, with endstage disease, dominated by the progressive LV dysfunction.

These limit the power of our conclusion that might not be extrapolated to other scenarios and should, therefore, be considered hypotheses generating.

\section{Conclusions}

Arrhythmogenesis in DCM is complex and derives from different mechanisms. The role of all possible contributing mechanisms, including fibrosis, inflammation, or genetics, is important, considering the actual difficulty in stratifying SCD risk. Our findings contribute to the better understanding of electric burden in DCM patients and reinforce, in part, the potential usefulness of genetic testing to improve the selection of candidates for ICD implantation.

Until the role of molecular diagnosis in the decision of ICD implantation is well established, the criteria to proceed to genetic testing maybe should not be too restrictive, confined to the presence of familial events or a particular phenotypic characteristic.

The use of broader gene panels in larger cohorts and collection of extended follow-up data are essential to achieve more definitive conclusions about the clinical usefulness of genetic tests in SCD risk stratification in DCM patients.

\section{Data Availability}

The data used to support the findings of this study are available from the corresponding author upon request.

\section{Conflicts of Interest}

The authors declare that there are no conflicts of interest regarding the publication of this article.

\section{Acknowledgments}

FATIMA (Portuguese study of familial dilated cardiomyopathy) investigators include Dr. ${ }^{\mathrm{a}}$ Ana Cristina Oliveira, Dr. ${ }^{a}$ Ana Lebreiro, Dr. ${ }^{a}$ Ana Rita Almeida, Dr. António Madureira, Dr. ${ }^{a}$ Aurora Andrade, Prof. ${ }^{a}$ Cristina Gavina, Prof. $^{a}$ Dulce Brito, Dr. Emanuel Correia, Prof. Lino Gonçalves, Prof. Luís Lopes, Dr. ${ }^{a}$ Maria Teresa Faria, Dr. ${ }^{a}$ Olga Azevedo, Prof. Rui Baptista, and Dr. ${ }^{\text {a }}$ Teresa Pinho. This work was supported by the Foundation for Science and Technology of Portuguese Government (PTDC/BIM-MEC/ 0650/2012).

\section{References}

[1] P. Ponikowski, A. A. Voors, S. D. Anker et al., "2016 ESC Guidelines for the diagnosis and treatment of acute and chronic heart failure," European Heart Journal, vol. 37, no. 27, pp. 2129-2200, 2016.

[2] E. Koutalas, E. Kanoupakis, and P. Vardas, "Sudden cardiac death in non-ischemic dilated cardiomyopathy: a critical appraisal of existing and potential risk stratification tools," International Journal of Cardiology, vol. 167, no. 2, pp. 335341, 2013.

[3] D. L. Packer, J. M. Prutkin, A. S. Hellkamp et al., "Impact of implantable cardioverter-defibrillator, amiodarone, and placebo on the mode of death in stable patients with heart failure: analysis from the sudden cardiac death in heart failure trial," Circulation, vol. 120, no. 22, pp. 2170-2176, 2009.

[4] L. Køber, J. J. Thune, J. C. Nielsen et al., "Defibrillator implantation in patients with nonischemic systolic heart failure," New England Journal of Medicine, vol. 375, no. 13, pp. 1221-1230, 2016.

[5] I. A. W. van Rijsingen, E. Arbustini, P. M. Elliott et al., "Risk factors for malignant ventricular arrhythmias in lamin A/C mutation carriers," Journal of the American College of Cardiology, vol. 59, no. 5, pp. 493-500, 2012.

[6] M. J. Ackerman, S. G. Priori, S. Willems et al., "HRS/EHRA expert consensus statement on the state of genetic testing for the channelopathies and cardiomyopathies," Heart Rhythm, vol. 8, no. 8, pp. 1308-1339, 2011.

[7] M. A. Burke, S. A. Cook, J. G. Seidman, and C. E. Seidman, "Clinical and mechanistic insights into the genetics of cardiomyopathy," Journal of the American College of Cardiology, vol. 68, pp. 2871-2886, 2016.

[8] S. Richards, N. Aziz, S. Bale et al., "Standards and guidelines for the interpretation of sequence variants: a joint consensus recommendation of the American College of medical genetics and genomics and the association for molecular Pathology," Genetics in Medicine, vol. 17, no. 5, pp. 405-423, 2015.

[9] S. G. Priori, C. Blomström-Lundqvist, A. Mazzanti et al., "2015 ESC Guidelines for the management of patients with ventricular arrhythmias and the prevention of sudden cardiac 
death," European Heart Journal, vol. 36, no. 41, pp. 27932867, 2015.

[10] P. Elliott, B. Andersson, E. Arbustini et al., "Classification of the cardiomyopathies: a position statement from the european society of Cardiology working group on myocardial and pericardial diseases," European Heart Journal, vol. 29, no. 2, pp. 270-276, 2008.

[11] E. Martins, J. Silva-Cardoso, M. Bicho et al., "Portuguese study of familial dilated cardiomyopathy: the FATIMA study," Revista Portuguesa de Cardiologia, vol. 27, no. 9, pp. 1029-1042, 2008.

[12] L. Mestroni, B. Maisch, W. J. McKenna et al., "Guidelines for the study of familial dilated cardiomyopathies," European Heart Journal, vol. 20, no. 2, pp. 93-102, 1999.

[13] P. C. Ng and S. Henikoff, "SIFT: predicting amino acid changes that affect protein function," Nucleic Acids Research, vol. 31, no. 13, pp. 3812-3814, 2003.

[14] J. M. Schwarz, D. N. Cooper, M. Schuelke, and D. Seelow, "MutationTaster2: mutation prediction for the deepsequencing age," Nature Methods, vol. 11, no. 4, pp. 361-362, 2014.

[15] I. A. Adzhubei, S. Schmidt, L. Peshkin et al., "A method and server for predicting damaging missense mutations," Nature Methods, vol. 7, no. 4, pp. 248-249, 2010.

[16] M. Lek, K. J. Karczewski, E. V. Minikel et al., "Analysis of protein-coding genetic variation in 60,706 humans," Nature, vol. 536, no. 7616, pp. 285-291, 2016.

[17] C. Genomes Project, A. Auton, L. D. Brooks et al., "A global reference for human genetic variation," Nature, vol. 526, no. 7571, pp. 68-74, 2015.

[18] S. T. Sherry, M. H. Ward, M. Kholodov et al., "dbSNP: the NCBI database of genetic variation," Nucleic Acids Research, vol. 29, no. 1, pp. 308-311, 2001.

[19] P. D. Stenson, E. V. Ball, M. Mort et al., "Human gene mutation database $\left(\mathrm{HGMD}^{\circledR}\right)$ : 2003 update," Human Mutation, vol. 21, no. 6, pp. 577-581, 2003.

[20] M. J. Landrum, J. M. Lee, M. Benson et al., "ClinVar: improving access to variant interpretations and supporting evidence," Nucleic Acids Research, vol. 46, no. D1, pp. D1062-D1067, 2018.

[21] I. F. A. C. Fokkema, P. E. M. Taschner, G. C. P. Schaafsma, J. Celli, J. F. J. Laros, and J. T. den Dunnen, "LOVD v.2.0: the next generation in gene variant databases," Human Mutation, vol. 32, no. 5, pp. 557-563, 2011.

[22] N. Sun, M. Yazawa, J. Liu et al., "Patient-specific induced pluripotent stem cells as a model for familial dilated cardiomyopathy," Science Translational Medicine, vol. 4, article 130ra47, 2012.

[23] N. Campbell, G. Sinagra, K. L. Jones et al., "Whole exome sequencing identifies a troponin $\mathrm{T}$ mutation hot spot in familial dilated cardiomyopathy," PLoS One, vol. 8, no. 10, Article ID e78104, 2013.

[24] T. Tobita, S. Nomura, T. Fujita et al., "Genetic basis of cardiomyopathy and the genotypes involved in prognosis and left ventricular reverse remodeling," Scientific Reports, vol. 8, p. 1998, 2018.

[25] M. Dal Ferro, D. Stolfo, A. Altinier et al., "Association between mutation status and left ventricular reverse remodelling in dilated cardiomyopathy," Heart, vol. 103, no. 21, pp. 1704-1710, 2017.

[26] J. Haas, K. S. Frese, B. Peil et al., "Atlas of the clinical genetics of human dilated cardiomyopathy," European Heart Journal, vol. 36, no. 18, pp. 1123-1135, 2015.
[27] E. Kayvanpour, F. Sedaghat-Hamedani, A. Amr et al., "Genotype-phenotype associations in dilated cardiomyopathy: meta-analysis on more than 8000 individuals," Clinical Research in Cardiology, vol. 106, no. 2, pp. 127-139, 2017.

[28] M. Merlo, A. Cannatà, M. Gobbo, D. Stolfo, P. M. Elliott, and G. Sinagra, "Evolving concepts in dilated cardiomyopathy," European Journal of Heart Failure, vol. 20, no. 2, pp. 228-239, 2018.

[29] P. A. van der Zwaag, I. A. W. van Rijsingen, A. Asimaki et al., "Phospholamban R14del mutation in patients diagnosed with dilated cardiomyopathy or arrhythmogenic right ventricular cardiomyopathy: evidence supporting the concept of arrhythmogenic cardiomyopathy," European Journal of Heart Failure, vol. 14, no. 11, pp. 1199-1207, 2012.

[30] A. Anastasakis and C. Basso, "“Primary" dilated hearts," International Journal of Cardiology, vol. 257, pp. 366-370, 2018.

[31] G.-S. Liu, A. Morales, E. Vafiadaki et al., "A novel human R25C-phospholamban mutation is associated with superinhibition of calcium cycling and ventricular arrhythmia," Cardiovascular Research, vol. 107, no. 1, pp. 164-174, 2015.

[32] M. G. Posch, A. Perrot, C. Geier et al., "Genetic deletion of arginine 14 in phospholamban causes dilated cardiomyopathy with attenuated electrocardiographic R amplitudes," Heart Rhythm, vol. 6, no. 4, pp. 480-486, 2009.

[33] K. Haghighi, F. Kolokathis, A. O. Gramolini et al., "A mutation in the human phospholamban gene, deleting arginine 14, results in lethal, hereditary cardiomyopathy," Proceedings of the National Academy of Sciences, vol. 103, no. 5, pp. 1388-1393, 2006.

[34] C. J. M. van Opbergen, M. Delmar, and T. A. B. van Veen, "Potential new mechanisms of pro-arrhythmia in arrhythmogenic cardiomyopathy: focus on calcium sensitive pathways," Netherlands Heart Journal, vol. 25, no. 3, pp. 157-169, 2017.

[35] B. P. Halliday, A. Gulati, A. Ali et al., "Association between midwall late gadolinium enhancement and sudden cardiac death in patients with dilated cardiomyopathy and mild and moderate left ventricular systolic dysfunction," Circulation, vol. 135, no. 22, pp. 2106-2115, 2017.

[36] R. E. Hershberger, M. M. Givertz, C. Y. Ho et al., "Genetic evaluation of cardiomyopathy-A heart failure society of America practice guideline," Journal of Cardiac Failure, vol. 24, no. 5, pp. 281-302, 2018. 


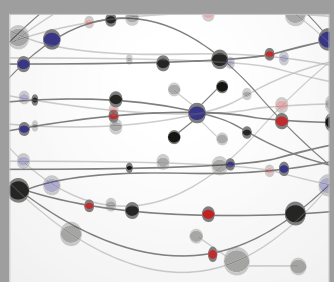

The Scientific World Journal
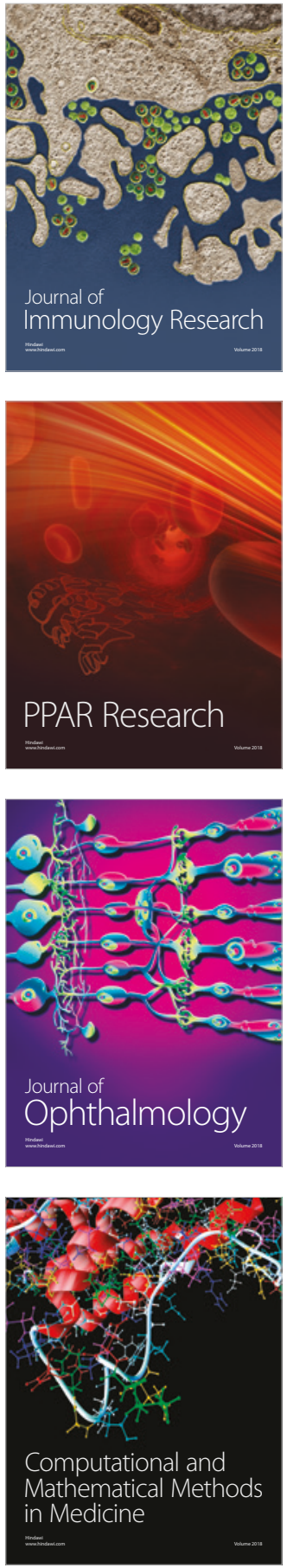

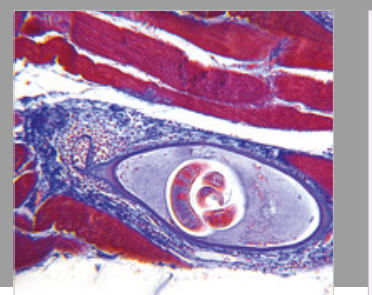

Gastroenterology Research and Practice

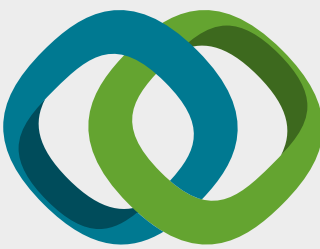

\section{Hindawi}

Submit your manuscripts at

www.hindawi.com
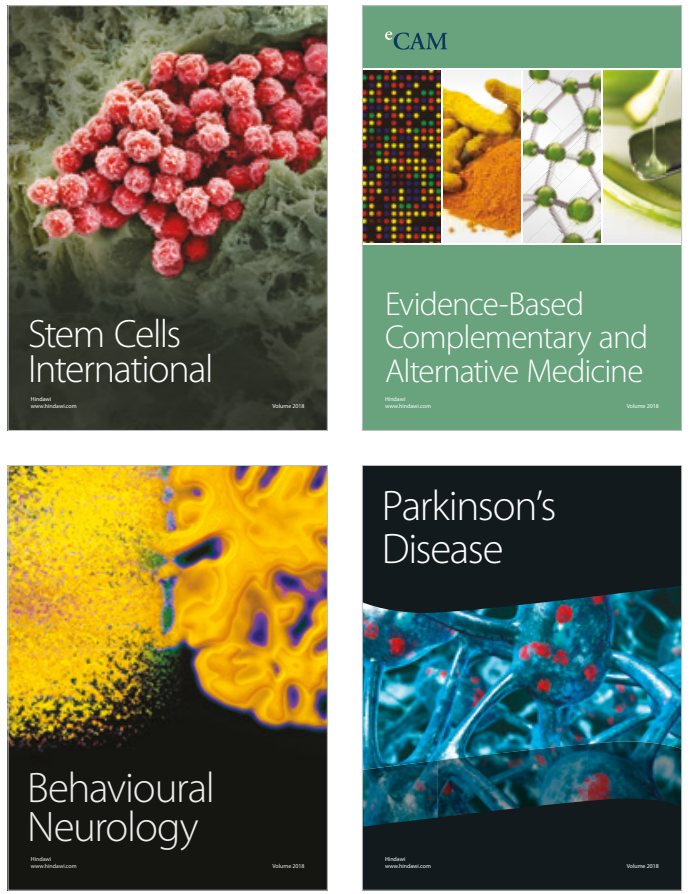

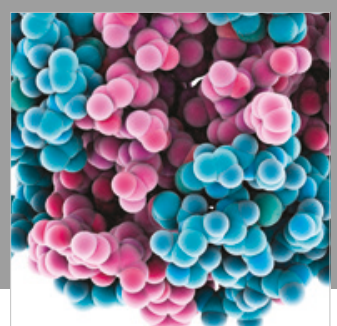

ournal of

Diabetes Research

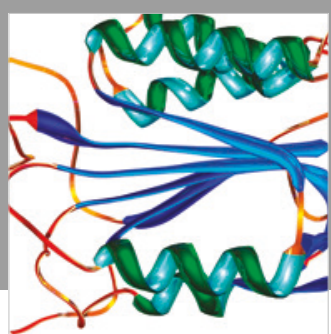

Disease Markers
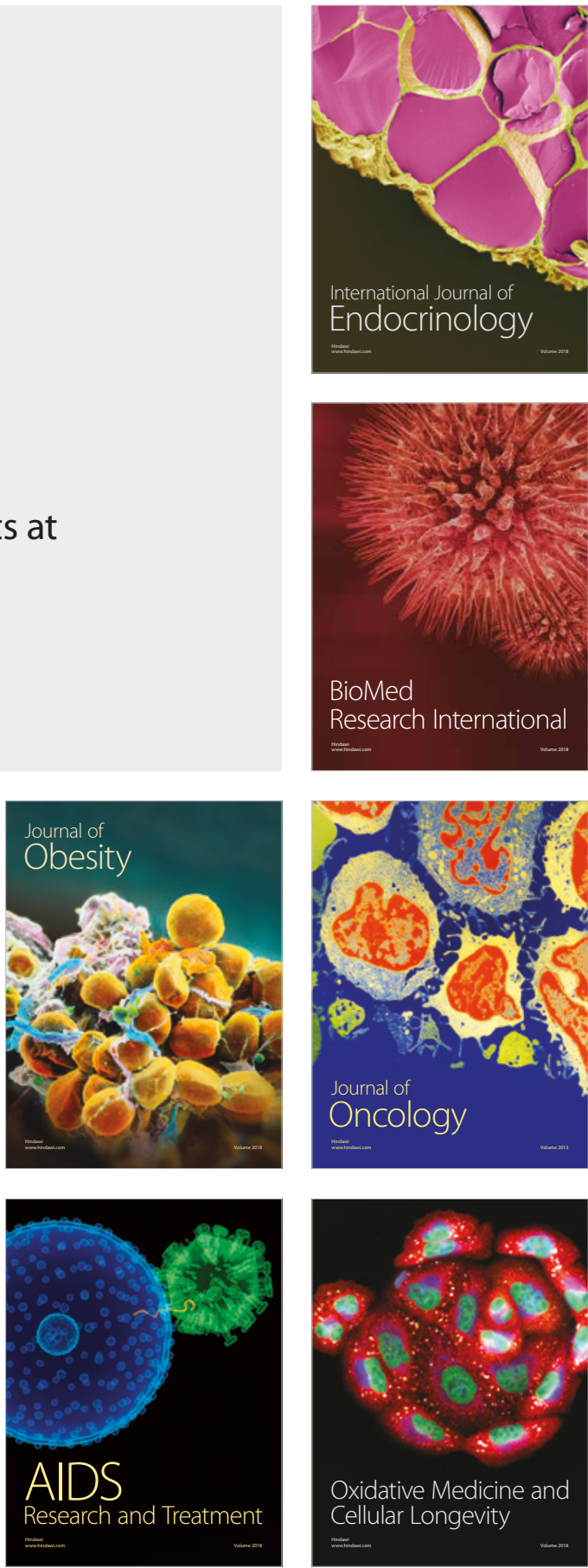